\title{
Invasive fungal infections
}

\section{William Hope, ${ }^{1,2}$ professor; Pavithra Natarajan, ${ }^{2}$ doctor; Lynsey Goodwin, ${ }^{2}$ doctor}

${ }^{1}$ University of Liverpool, UK; ${ }^{2}$ Royal Liverpool Hospital, UK

Invasive fungal infections continue to extract a significant toll on human health and to be associated with excessive morbidity and mortality. The clinical challenges posed by these organisms are many and protean. Patients who are at risk of invasive fungal infections often have multiple comorbidities. Diagnostic tests are slow, often with low sensitivity and/or specificity and are expensive. Furthermore, there are limited therapeutic options. Many antifungal agents have significant drug-related toxicity, drug-drug interactions and are extremely expensive.

In a tertiary care setting that contains a large number of patients who are immunocompromised, a wide variety of fungal pathogens will be encountered. These will ordinarily require specialist laboratory and clinical skills to aid in the diagnosis and management. The most common medically important fungal pathogens in resource-rich healthcare settings are Aspergillus spp and Candida spp. From a global perspective, Cryptococcus neoformans is a leading cause of infectious morbidity and mortality, with an estimated 600,000 deaths per year. ${ }^{1}$ Clinicians might encounter a wide range of relatively rare and esoteric pathogens belonging to Mucorales (eg Rhizopus spp), hyalohyphomycetes (eg Fusarium and Scedosporium spp) or phaeohyphomycetes (eg Alternaria spp and Cladophialophora bantiana). These organisms are frequently difficult to treat and require specialist consultation. A variety of endemic fungal diseases might also be encountered (eg histoplasmosis, blastomycosis, coccidioidomycosis and paracoccidioidomycosis), making a detailed travel and occupational history mandatory. In this article, we focus on the most common medically important fungal pathogens in the UK: Aspergillus and Candida spp.

\section{Invasive aspergillosis}

\section{Introduction}

Invasive aspergillosis (IA) is a leading cause of infectious morbidity and mortality in patients who are profoundly immunocompromised. The most common clinical contexts where IA is seen include profound and prolonged neutropenia, haematopoietic stem-cell transplantation and solid organ transplantation (especially lung transplantation). IA is also increasingly seen in patients who are critically ill on intensive care units ${ }^{2}$ and might also be seen in a variety of scenarios where there is significant underlying damage to the lung (eg near drowning or post influenza).

\section{Diagnosis}

IA is notoriously difficult to diagnose with any certainty. In the UK, most diagnoses are made on the basis of a computed tomography (CT) scan of the chest in a patient with appropriate risk factors (eg a patient who is profoundly immunocompromised with fever and/or other respiratory symptoms). Some radiological features are specific of invasive pulmonary aspergillosis (IPA): a nodule with surrounding attenuation (halo) is a key radiological sign. An air crescent sign is a late radiological sign resulting from organisation of a pulmonary sequestrum. More commonly, multiple nodules are the only radiological sign of infection. In reality, any radiological appearance can be seen (eg non-specific focal infiltrates, pleural effusion or pulmonary infiltrates with cavitation), which poses a significant diagnostic challenge.

Specific microbiological evidence of IA can be difficult to acquire. In many centres, serum galactomannan is increasingly used to establish a diagnosis of IA. Galactomannan is a large-molecularweight polysaccharide that is secreted by Aspergillus hyphae (Fig 1). The sensitivity of this test can be compromised by mouldactive prophylaxis or specific antifungal treatment (eg itraconazole) and also varies according to whether the patient has underlying neutropenia. ${ }^{3}$ The diagnostic performance of this test depends on the incidence and case mix. A bronchoalveolar lavage (BAL) might not be possible because

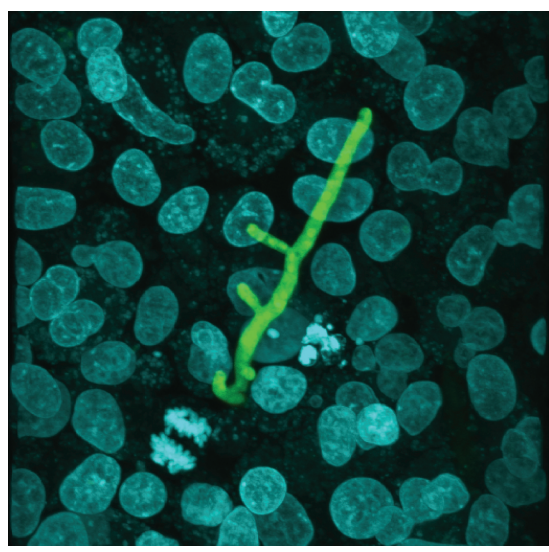

Fig 1. A confocal image of Aspergillus fumigatus invading through endothelial cells. Nuclei of the endothelial cells are stained with 4',6-diamidino-2-phenylindole (DAPI). The angular dichotomous branching that is typical of Aspergillus spp is evident. Actively growing hyphae liberate galactomannan (among a multitude of secondary metabolites) that can be used for the diagnosis of IA, as well as for monitoring the effect of antifungal therapy.

of poor respiratory reserve or other comorbidities. Positive cultures are only seen in approximately $50 \%$ of cases. ${ }^{3}$ Galactomannan in BAL fluid is increasingly used to establish a diagnosis of IPA, although there is some uncertainty about the precise cut-off that should be used to secure a diagnosis (a reading of $>1$ is often used).

\section{Treatment}

The treatment of IA is complex and requires specialist advice and consultation. In addition to choosing an appropriate antifungal agent and regimen, careful consideration should be given to modifying host factors (eg minimising immunosuppression), as well as providing adjunctive care (eg granulocyte colony-stimulating factor [G-CSF]). Surgery might be necessary to obtain a cure or at least debulk disease to enable successful medical therapy. The indications for surgery are not well defined, but include extensive surgically resectable disease near vital structures (eg mediastinum or great vessels).

Several first-line agents can be used to treat IA (Table 1). The choice largely rests between voriconazole and liposomal amphotericin B. Voriconazole is superior to amphotericin B deoxycholate for treatment of IA in 


\section{Key points}

Invasive fungal infections are often rapidly life threatening and require prompt diagnosis and therapy as well as specialist consultation

First-line agents for the treatment of invasive aspergillosis include voriconazole and liposomal amphotericin B

Therapeutic drug monitoring should be considered as a routine adjunct when using voriconazole and posaconazole

The echinocandins (caspofungin, micafungin and anidulafungin) are first-line agents for the treatment of invasive candidiasis

Patients with invasive candidiasis should have prompt medical therapy and a careful search for metastatic infection

KEY WORDS: Fungus, invasive, Aspergillus, Candida, antifungal

a wide range of clinical scenarios. ${ }^{4}$ Amphotericin B deoxycholate is now not widely available in the UK. Voriconazole is available as an intravenous (IV) and oral preparation, which enables induction therapy followed by longer-term consolidation therapy. A dose of $6 \mathrm{mg} / \mathrm{kg}$ IV every $12 \mathrm{~h}$ for two dosages should be given, followed by $4 \mathrm{mg} / \mathrm{kg}$ every $12 \mathrm{~h}$ as IV maintenance. The dose for oral therapy is $200 \mathrm{mg}$ every $12 \mathrm{~h}$, with the possibility of increasing this to $300 \mathrm{mg}$ every $12 \mathrm{~h}$ if clinically indicated. Voriconazole is certainly the drug of choice for treatment of cerebral aspergillosis, which is a devastating complication of disseminated disease. ${ }^{5}$ Increasing evidence suggests that therapeutic drug monitoring should be performed routinely. ${ }^{6}$ A trough concentration of $1 \mathrm{mg} / \mathrm{l}$ is a reasonable target, but higher concentration targets can be used if clinically indicated (eg central nervous system infection). An upper concentration target of $5-6 \mathrm{mg} / \mathrm{l}$ is recommended to minimise the probability of drug-related toxicity. ${ }^{6}$ Voriconazole exhibits well-described toxicities that are related to serum concentrations, including elevated liver function tests, confusion and hallucinations. Other short-term (and reversible) adverse effects include photopsia (a perception of flashing flights), which occurs in approximately $30 \%$ of patients, and is transitory and fully reversible. Longer-term toxicities include photosensitivity, which has been further complicated in some patients by the development of skin cancers. Consequently, patients should receive specific advice about protecting themselves from the sun, including the routine application of sunscreen. An alternative first-line agent for treatment of IA is liposomal amphotericin B (Table 1). A clinical trial of patients with IA suggested that the effect of a dose of $3 \mathrm{mg} / \mathrm{kg} /$ day is comparable to 10 $\mathrm{mg} / \mathrm{kg} /$ day. ${ }^{7}$ A patient failing $3 \mathrm{mg} / \mathrm{kg}$ should have their antifungal therapy changed or another antifungal agent added. Liposomal amphotericin B has the advantage of having a broader-spectrum of activity compared with voriconazole, including activity against the Mucorales and triazole-resistant Aspergillus spp that are increasingly seen in Europe and other parts of the world. Liposomal amphotericin B causes acute infusional toxicity ${ }^{8}$ (manifests as any combination of chest pain, flank pain, leg pain, abdominal pain, dyspnoea, hypoxia, urticaria or flushing) as well as nephrotoxicity (manifests as decreased glomerular filtration rate or tubular toxicity with hypokalaemia and/or hypomagnesaemia). ${ }^{7}$

There are relatively few alternative agents or approaches for treatment of IA. The results of several clinical trials are pending and might provide some additional therapeutic avenues. The outcome of patients receiving a combination of voriconazole and anidulafungin vs voriconazole alone will be published in the near future. Furthermore, the results of a new triazole, isavuconazole vs voriconazole, will be available, which might provide an alternative agent for the treatment of IA. The echinocandins are generally not advocated as monotherapy for the primary treatment of IA, although they might be useful for salvage therapy. ${ }^{9}$ Similarly, posaconazole is not used for primary therapy, but might have a useful role for salvage therapy or longerterm consolidation therapy. ${ }^{10}$

\section{Invasive candidiasis}

Invasive candidiasis remains a significant cause of morbidity and mortality for some patient groups. Candidaemia is mostly seen in patients who are critically ill and nonneutropaenic. There are progressively fewer cases in patients with neutropenia, which is probably related to the better use of antifungal prophylaxis and other supportive measures in these patients. Risk factors include the presence of an intravenous catheter and the use of total parenteral nutrition. The most common cause of invasive candidiasis is Candida albicans. Other leading causes include Candida glabrata (often exhibits reduced susceptibility to fluconazole), Candida parapsilosis (exhibits reduced susceptibility to echinocandins), Candida krusei (resistant to fluconazole) and Candida tropicalis (leading cause of invasive candidiasis in India).

\section{Diagnosis}

Invasive candidiasis is mostly diagnosed using standard microbiological techniques. Blood cultures are the mainstay of the diagnosis, even though the sensitivity is known to be approximately $50-60 \% .^{11}$ Invasive candidiasis can also be diagnosed using samples taken from normally sterile sites (eg pancreatic bed). Some serological tests are available to aid in the diagnosis. Perhaps the most promising is $1,3-\beta-\mathrm{D}$ glucan, for which there is a large amount of evidence. ${ }^{11}$ Unfortunately, this test is relatively difficult to perform and is still not widely available in many centres.

\section{Treatment}

The management of invasive candidiasis requires prompt initiation of antifungal therapy. Candida spp should never be dismissed as contaminants when isolated from blood cultures or other ordinarily sterile site. Relatively small delays (circa 12-24 h) in the initiation of antifungal therapy result in excessive mortality. ${ }^{12}$ Important additional 
therapeutic considerations include the removal (and potential replacement) of an intravenous catheter. A thorough search should be made for occult metastatic foci of infection. Candida endophthalmitis is a serious and potentially sight-threatening complication of disseminated infection that requires specialised management. Consequently, an ophthalmological examination should be performed on all patients with candidaemia and an ophthalmological opinion sought if there is any evidence or concern of endophthalmitis.

Several agents can be used to treat invasive candidiasis (Table 2). Recent evidence and treatment guidelines suggest that the echinocandins should be considered as first-line agents for initial treatment of invasive candidiasis. ${ }^{13,14}$ There is no clear evidence to distinguish caspofungin $(70 \mathrm{mg} / \mathrm{day}$ as a loading dose followed by $50 \mathrm{mg} /$ day), ${ }^{15}$ micafungin $(100 \mathrm{mg} / \text { day })^{16}$ and anidulafungin $(200 \mathrm{mg} /$ day as a loading dose followed by $100 \mathrm{mg} /$ day) ${ }^{17}$ for the treatment of candidaemia and invasive candidiasis: the spectrum of microbiological activity and clinical response rates in clinical trials of invasive candidiasis are comparable. The echinocandins have the distinct advantage of being safe to use in patients with renal and hepatic impairment. ${ }^{18}$ Furthermore, they have few adverse effects and few clinically significant drug-drug interactions. Nevertheless, one potential issue with their routine use is the treatment of $C$ parapsilosis, which consistently demonstrates relatively high minimum inhibitory concentrations as a result of differences in the echinocandin target site (FKS1 gene); favourable clinical outcomes in invasive candidiasis caused by $C$ parapsilosis were lower with anidulafungin, but not statistically inferior to fluconazole. ${ }^{17}$ Therefore, the decision to use an echinocandin for $C$ parapsilosis depends on the clinical context. The echinocandins are probably the drug of choice for the treatment of $C$ glabrata, which exhibits reduced susceptibility or even frank resistance to fluconazole. The echinocandins can also be used for the treatment of $C$ krusei, which is inherently resistant to fluconazole.

Liposomal amphotericin B at $3 \mathrm{mg} / \mathrm{kg}$ can be used to treat invasive candidiasis. ${ }^{19} \mathrm{~A}$ clinical trial suggested that this agent produces response rates that are comparable to

Table 1. Therapeutic options for the treatment of invasive aspergillosis.

\begin{tabular}{|c|c|c|}
\hline Therapy & Regimen & Comments \\
\hline \multicolumn{3}{|l|}{ Primary therapy } \\
\hline Voriconazole & $\begin{array}{l}6 \mathrm{mg} / \mathrm{kg} \text { IV Q12h } \times 2 \text { dosages, followed by } 4 \mathrm{mg} / \mathrm{kg} \\
\text { Q12h IV; oral therapy } 200 \mathrm{mg} \text { Q12h, with option to } \\
\text { increase to } 300 \mathrm{mg} \text { Q } 12 \mathrm{~h}\end{array}$ & $\begin{array}{l}\text { Therapeutic drug monitoring should be considered with dosage } \\
\text { adjustment to keep trough concentrations }>1 \mathrm{mg} / \mathrm{l} \text { and }<6 \mathrm{mg} / \mathrm{l}\end{array}$ \\
\hline Liposomal amphotericin B & $3 \mathrm{mg} / \mathrm{kg} /$ day IV & Monitor renal function \\
\hline \multicolumn{3}{|l|}{ Salvage therapy } \\
\hline Caspofungin & $70 \mathrm{mg}$ loading dose, then $50 \mathrm{mg} /$ day & \\
\hline Micafungin & 100-150 mg/day & $\begin{array}{l}\text { Not licensed for this indication; optimal dose for invasive } \\
\text { aspergillosis not known }\end{array}$ \\
\hline Posaconazole & $200 \mathrm{mg}$ Q6h followed by $400 \mathrm{mg}$ Q12h & $\begin{array}{l}\text { Therapeutic drug monitoring should be considered with dosage } \\
\text { adjustment to keep trough concentrations }>1 \mathrm{mg} / \mathrm{l}\end{array}$ \\
\hline
\end{tabular}

Table 2. Therapeutic options for the treatment of invasive candidiasis.

\begin{tabular}{|c|c|c|}
\hline Therapy & Regimen & Comments \\
\hline \multicolumn{3}{|l|}{ Primary therapy } \\
\hline Caspofungin & $70 \mathrm{mg}$ as a loading dose, then $50 \mathrm{mg} / \mathrm{day}$ & \\
\hline Micafungin & $100-150 \mathrm{mg} /$ day & No difference in clinical outcomes between 100 and $150 \mathrm{mg} /$ day \\
\hline Anidulafungin & $200 \mathrm{mg}$ as a loading dose followed by $100 \mathrm{mg} /$ day & \\
\hline Liposomal amphotericin B & $3 \mathrm{mg} / \mathrm{kg} /$ day IV & Monitor renal function \\
\hline Fluconazole & $400-800 \mathrm{mg} /$ day & Increasingly, echinocandins are preferred for induction therapy \\
\hline Voriconazole & $\begin{array}{l}6 \mathrm{mg} / \mathrm{kg} \text { IV Q12h } \times 2 \text { dosages, followed by } 4 \mathrm{mg} / \mathrm{kg} \\
\text { Q12h IV; oral therapy } 200 \mathrm{mg} \text { Q12h }\end{array}$ & $\begin{array}{l}\text { Therapeutic drug monitoring should be considered with dosage } \\
\text { adjustment to keep trough concentrations }>1 \mathrm{mg} / \mathrm{l} \text { and }<6 \mathrm{mg} / \mathrm{l}\end{array}$ \\
\hline \multicolumn{3}{|l|}{ Consolidation therapy } \\
\hline Fluconazole & $400-800 \mathrm{mg} /$ day & Switch to fluconazole when the patient is haemodynamically stable \\
\hline Voriconazole & $\begin{array}{l}6 \mathrm{mg} / \mathrm{kg} \text { IV Q12h } \times 2 \text { dosages, followed by } 4 \mathrm{mg} / \mathrm{kg} \\
\text { Q12h IV; oral therapy } 200 \mathrm{mg} \text { Q12h }\end{array}$ & $\begin{array}{l}\text { Therapeutic drug monitoring should be considered with dosage } \\
\text { adjustment to keep trough concentrations }>1 \mathrm{mg} / \mathrm{l} \text { and }<6 \mathrm{mg} / \mathrm{l} \text {; } \\
\text { consider for patients with Candida krusei and Candida glabrata }\end{array}$ \\
\hline
\end{tabular}


micafungin, although the rate of nephrotoxicity was predictably higher. Liposomal amphotericin B has an advantage of a broad spectrum of activity against medically important Candida spp. Fluconazole is still extensively used for the treatment of invasive candidiasis, is the agent of choice for consolidation therapy in most cases and can be used immediately after initial induction therapy with an echinocandin or liposomal amphotericin B. Potential exceptions include primary treatment for infections caused by $C$ glabrata and $C$ krusei. In these cases, voriconazole might be a suitable option, with all the caveats previously discussed for this compound when used for IA.

\section{Conclusions}

Invasive fungal infections continue to be a significant problem in a wide range of clinical contexts. There have been significant advances in therapeutic options for invasive infections caused by Aspergillus and Candida spp. Successful treatment of these opportunistic infections requires prompt diagnosis and aggressive therapy with antifungal agents.

\section{References}

1 Park BJ, Wannemuehler KA, Marston BJ et al. Estimation of the current global burden of cryptococcal meningitis among persons living with HIV/AIDS. Aids 2009;23:525-30.

2 Meersseman W, Vandecasteele SJ, Wilmer A et al. Invasive aspergillosis in critically ill patients without malignancy. Am J Respir Crit Care Med 2004;170:621-5.
3 Hope WW, Walsh TJ, Denning DW. Laboratory diagnosis of invasive aspergillosis. Lancet Infect Dis 2005;5:609-22.

4 Herbrecht R, Denning DW, Patterson TF et al. Voriconazole versus amphotericin B for primary therapy of invasive aspergillosis. N Engl J Med 2002;347:408-15.

5 Schwartz S, Ruhnke M, Ribaud P et al. Improved outcome in central nervous system aspergillosis, using voriconazole treatment. Blood 2005;106:2641-5.

6 Pascual A, Calandra T, Bolay S et al. Voriconazole therapeutic drug monitoring in patients with invasive mycoses improves efficacy and safety outcomes. Clin Infect Dis 2008;46:201-11.

7 Cornely OA, Maertens J, Bresnik M et al. Liposomal amphotericin B as initial therapy for invasive mold infection: a randomized trial comparing a high-loading dose regimen with standard dosing (AmBiLoad trial). Clin Infect Dis 2007;44:1289-97.

8 Roden MM, Nelson LD, Knudsen TA et al. Triad of acute infusion-related reactions associated with liposomal amphotericin B: analysis of clinical and epidemiological characteristics. Clin Infect Dis 2003;36:1213-20.

9 Maertens J, Raad I, Petrikkos G et al. Efficacy and safety of caspofungin for treatment of invasive aspergillosis in patients refractory to or intolerant of conventional antifungal therapy. Clin Infect Dis 2004;39:1563-71.

10 Walsh TJ, Raad I, Patterson TF et al. Treatment of invasive aspergillosis with posaconazole in patients who are refractory to or intolerant of conventional therapy: an externally controlled trial. Clin Infect Dis 2007;44:2-12.

11 Cuenca-Estrella M, Verweij PE, Arendrup MC et al. ESCMID* guideline for the diagnosis and management of Candida diseases 2012: diagnostic procedures. Clin Microbiol Infect 2012;18(suppl 7):9-18.
12 Morrell M, Fraser VJ, Kollef MH. Delaying the empiric treatment of candida bloodstream infection until positive blood culture results are obtained: a potential risk factor for hospital mortality. Antimicrob Agents Chemother 2005;49:3640-5.

13 Cornely OA, Bassetti M, Calandra T et al. ESCMID* guideline for the diagnosis and management of Candida diseases 2012: non-neutropenic adult patients. Clin Microbiol Infect 2012;18(suppl 7):19-37.

14 Ullmann AJ, Akova M, Herbrecht R et al. ESCMID* guideline for the diagnosis and management of Candida diseases 2012: adults with haematological malignancies and after haematopoietic stem cell transplantation (HCT). Clin Microbiol Infect 2012;18(suppl 7):53-67.

15 Mora-Duarte J, Betts R, Rotstein C et al. Comparison of caspofungin and amphotericin B for invasive candidiasis. $N$ Engl $J$ Med 2002;347:2020-9.

16 Pappas PG, Rotstein CM, Betts RF et al. Micafungin versus caspofungin for treatment of candidemia and other forms of invasive candidiasis. Clin Infect Dis 2007;45:883-93.

17 Reboli AC, Rotstein C, Pappas PG et al. Anidulafungin versus fluconazole for invasive candidiasis. N Engl J Med 2007;356:2472-82.

18 Denning DW, Hope WW. Therapy for fungal diseases: opportunities and priorities. Trends Microbiol 2010;18:195-204.

19 Kuse ER, Chetchotisakd P, da Cunha CA et al. Micafungin versus liposomal amphotericin B for candidaemia and invasive candidosis: a phase III randomised doubleblind trial. Lancet 2007;369:1519-27.

\section{Address for correspondence:}

Prof W Hope, Antimicrobial

Pharmacodynamics and Therapeutics, 1.09 Sherrington Building, Ashton

Street, Liverpool L69 3GE

Email: william.hope@liverpool.ac.uk 